Le genre de la maladie : pratiques, discours, textes et représentations

\title{
Maladie individuelle, crise du couple et maux collectifs dans le roman Du stirbst nicht de Kathrin Schmidt : des transitions de l'après-1989 au prisme du genre
}

Sibylle GOEPPER

\section{OpenEdition}

\section{Journals}

\section{Electronic version}

URL: http://journals.openedition.org/transtexts/665

DOI: $10.4000 /$ transtexts.665

ISSN: 2105-2549

Publisher

Gregory B. Lee

\section{Electronic reference}

Sibylle GOEPPER, «Maladie individuelle, crise du couple et maux collectifs dans le roman Du stirbst nicht de Kathrin Schmidt : des transitions de l'après-1989 au prisme du genre », Transtext(e)s

Transcultures 跨文本跨文化 [Online], 11 | 2016, Online since 30 June 2017, connection on 30 April 2019. URL : http://journals.openedition.org/transtexts/665 ; DOI : 10.4000/transtexts.665

This text was automatically generated on 30 April 2019.

(C) Tous droits réservés 


\title{
Maladie individuelle, crise $\mathrm{du}$ couple et maux collectifs dans le roman Du stirbst nicht de Kathrin Schmidt : des transitions de l'après-1989 au prisme du genre
}

\author{
Sibylle GOEPPER
}

\author{
La maladie est la zone d'ombre de la vie, un \\ territoire auquel il coûte cher d'appartenir. En \\ naissant, nous acquérons une double nationalité \\ qui relève du royaume des bien-portants comme \\ de celui des malades. Et bien que nous préférions \\ tous présenter le bon passeport, le jour vient où \\ chacun de nous est contraint, ne serait-ce qu'un \\ court moment, de se reconnaitre citoyen de l'autre \\ contrée. Susan Sontag, 1972
}

1 Si cette étude se penche sur le roman Du stirbst nicht ("Tu ne vas pas mourir », 2009) de l'écrivaine Kathrin Schmidt, c'est d'une part parce qu'il y est question de maladie, et plus exactement d'un accident cérébral et de ses suites, d'autre part parce que ce thème est abordé par une femme - du moins si l'on s'en tient au sexe biologique de la romancière. Ce choix offre ensuite un contrepoint aux contributions civilisationnelles de ce dossier, qui donnent largement la parole aux hommes, et s'inscrit dans le cadre d'un débat général : celui sur la soi-disant écriture "féminine ». ${ }^{1}$ L'intérêt porté au roman de cette écrivaine est-allemande donne en outre la possibilité de mobiliser simultanément les outils $\mathrm{du}$ genre et différents paramètres, et d'adopter ainsi une approche intersectionnelle (seule à même de rendre compte des textes de manière suffisamment différenciée selon l'auteure de cet article), autrement dit une perspective qui tienne compte pour l'analyse de la génération, du lieu de socialisation, de l'origine et de la 
trajectoire sociale, mais aussi du contexte historique, politique et culturel dans lequel l'œuvre se situe.

2 Les femmes artistes socialisées en République démocratique allemande (RDA) avant 1990 offrent une entrée d'autant plus intéressante pour le sujet qui nous occupe que l'État estallemand, conformément à la pensée des socialistes Marx, Engels et Bebel dont il se réclame, proclame l'égalité entre hommes et femmes dès la Constitution de 1949 et s'appuie dans son discours sur une vision universaliste et égalitaire des citoyennes et citoyens. ${ }^{2}$ Si l'on y ajoute qu'en théorie, la socialisation socialiste, en particulier dans l'Allemagne de l'Est d'après 1945, est fondée sur les valeurs d'antifascisme, de pacifisme et d'anti-impérialisme, tous les éléments d'une rupture avec le modèle « militaro-viril » de l'avant-guerre semblent réunis afin qu'advienne, avec «l'Homme nouveau » appelé de ses vœux par le communisme révolutionnaire, « la Femme nouvelle ».

3 Le suspense ne sera pas entretenu plus longtemps : évidemment, il s'agit là de quelquesuns des mythes fondateurs de l'État socialiste, le terme mythe étant entendu ici au sens de "grand récit », servant de ciment à la communauté, mais aussi de "légende », dont il convient de passer les ressorts au crible de la critique. En conclure pour autant que les Allemandes de l'Est connaissent une socialisation de genre similaire à celle des citoyennes de l'Ouest à la même époque n'en serait pas moins erroné. L'historienne Anna Kaminsky montre ainsi comment la nécessité d'intégrer les femmes à la population active est effectivement à l'origine de mesures progressistes dans le domaine du droit de la famille et social. ${ }^{3}$ Les questions : "Qu'est-ce que le socialisme a fait au genre, comment a-t-il modifié les rapports de genre? ? $\aleph^{4}$ posées par Sandrine Kott et Françoise Thébaud en 2015 paraîssent donc particulièrement stimulantes.

4 Nous proposons de nous interroger à notre tour sur la persistance et la nature d'une identité de genre au sein de la société socialiste, mais cette fois du point de vue des représentations, et plus précisément du traitement littéraire de la maladie. Nous nous pencherons pour ce faire sur une œuvre fictive, parue presque 20 ans après la réunification allemande. Une première remarque est que l'on n'est pas chez K. Schmidt dont le personnage est victime d'une rupture d'anévrisme - en présence d'un mal typiquement "féminin " , d'où l'hypothèse que son roman véhicule, peut-être, des représentations du lien entre genre et maladie autre que celles mises en évidence pour la tradition littéraire d'Europe de l'Ouest. ${ }^{6}$

5 Du stirbst nicht raconte le retour à la vie d'Helene Wesendahl, frappée par un accident cérébral à l'âge de 44 ans. Sa reprise de conscience ainsi que sa convalescence, étalées sur plusieurs mois, servent de fil conducteur à un récit qui croise des strates temporelles distinctes. Celles-ci ne sont pas présentées de façon linéaire, mais se superposent et s'enchevêtrent tout au long du roman; néanmoins, le découpage en chapitres fait apparaître des centres de gravité : les premiers, au présent, retracent l'immédiat après rupture d'anévrisme, les chapitres centraux évoquent le passé plus lointain en RDA, les derniers se focalisent sur les relations amoureuses du personnage principal. Enfin, en parallèle du travail de reconstruction physique s'enclenche un processus mémoriel à la fois sur le plan individuel, celui d'Helene Wesendahl, et collectif, par le biais de l'évocation de l'avant et de l'après chute du Mur de 1989.

6 Au moment d'aborder l'étude de ce texte, il convient de revenir également sur le genre, cette fois au sens de genre littéraire, auquel il appartient : outre le fait que K. Schmidt a été elle-même victime d'une rupture d'anévrisme en 2002, de nombreux éléments 
suggèrent une forte proximité entre l'auteure et son personnage : les cinq enfants, la vie dans une commune attenante à Berlin (Karlshorst au lieu de Mahlsdorf) ou encore l'activité en tant que psychologue dans un service pédiatrique du temps de la RDA, puis en tant qu'écrivaine indépendante après la réunification. J'ai montré ailleurs que $\mathrm{K}$. Schmidt, à l'instar d'autres auteurs de sa génération, effectuait avec cet ouvrage une transition esthétique qui la menait du genre lyrique au genre autofictionnel tel qu'il a été défini par Philippe Gasparini. ${ }^{7}$ Certaines de ses spécificités doivent être rappelées ici : d'une part, le caractère partiel du pacte référentiel conclu avec le lecteur, avec pour conséquence que si certains éléments semblent avérés, il en est d'autres qui se situent clairement du côté de la fiction; d'autre part, la dimension clairement plus historique de la variante « est-allemande » de l'autofiction en comparaison des formes que l'on connait en RFA ou en France, thèse également validée par la micro-analyse de Du stirbst nicht dans ce précédent travail. ${ }^{8}$

C'est à ces réflexions de départ que nous souhaiterions ajouter une perspective de genre, en posant quelques-unes des questions suivantes: les identités de genre ont-elles une importance dans l'économie du roman, correspondent-elles aux identités traditionnelles et hégémoniques? Que nous apprend-on des rapports de genre en RDA et dans l'Est de l'Allemagne aujourd'hui ? La narration de la pathologie se fait-elle depuis un point de vue " genré »? Enfin, l'écriture mise en œuvre peut-elle être qualifiée de « féminine » dans le sens évoqué plus haut?

8 Nous étudierons d'abord le texte de K. Schmidt sous l'angle de la séquelle : l'aphasie, pour commencer, qui condamne la patiente à une forme de claustration entre corps et conscience. Une deuxième partie s'attachera au phénomène de l'amnésie qui la contraint à reconstruire sa propre biographie avant et après 1989: nous y analyserons les symptômes de l'effritement progressif du système, de ses conséquences sur la population et du trauma collectif qui a suivi la disparition de la RDA. Dans un troisième temps, nous nous pencherons sur les effets salutogènes de la crise qui touche le couple, les amours ainsi que l'écriture. Ces thèmes seront abordés sous l'angle d'une "queerisation » et d'une hybridation fécondes du fond et de la forme.

\section{Aphasie : entre corps et conscience}

Les premières pages du roman s'ouvrent sur le retour progressif à la conscience d'Helene Wesendahl après une rupture d'anévrisme survenue quelques semaines plus tôt. Celui-ci se produit dans un premier temps par le biais de son appareil sensoriel auditif : elle perçoit des bruits et des voix, les phrases prononcées sont signalées en italique, mais ne sont pas attribuées à des personnes identifiées car elle ne voit rien. Ses yeux sont pourtant très présents. Tantôt ouverts, tantôt fermés, ils constituent un rideau naturel qui, sur le plan de la narration, permet de faire la transition entre les différentes séquences. Durant ces premières pages, le texte se compose de blocs très courts qui reflètent une capacité de perception et d'appréhension de la réalité fortement réduite, mais sans doute aussi, plus symboliquement, la fragmentation d'une vie qui a volé en éclat.

10 Bien que le récit soit mené à la troisième personne, censée créer une certaine distance entre narrateur et personnage, nous sommes immergés dans la conscience d'Helene. Cette perspective correspond au « régime d'objectivation subjectivée » défini par Thierry Groensteen: «Nous voyons les personnages de l'extérieur mais à la façon dont eux- 
mêmes perçoivent le monde et s'y projettent $"^{9}$. Le lecteur ne dispose pas de plus d'informations que la patiente, il est témoin des associations d'idées qu'elle génère, associations qui, lorsqu'elle tente d'intégrer ce qui se passe dans son environnement, sont souvent erronées : ainsi le cliquetis des appareils médicaux lui rappelle-t-il le bruit des

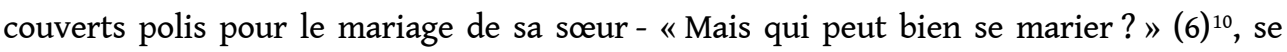
demande-t-elle. Sa difficulté à établir les connexions les plus élémentaires transparaît aussi dans les (fausses) hypothèses émises à propos des yeux rougis de son mari : souffre$\mathrm{t}$-il d'irritation oculaire ou pleure-t-il, comme lors de son dernier accouchement, parce qu'elle vient d'avoir un enfant?

11 L'absence de repères dans l'espace et dans le temps est quasi totale : Helene ignore où elle est, décrit au présent des scènes qui se sont produites dans le passé et n'est jamais entièrement sûre qu'un événement s'est bel et bien produit. Longtemps, les frontières entre rêve et réalité sont abolies, l'existence semblant flotter à mi-chemin entre sommeil diurne et nocturne, coma et narcose. Le cadre spatio-temporel n'est finalement rétabli qu'à la fin du premier chapitre, lorsque nous apprenons, en même temps qu'elle, qu'elle se trouve depuis trois semaines en soins intensifs.

K. Schmidt puise à la source de l'expérience biographique pour offrir une clinique certes littérarisée, mais néanmoins précise de la tabula rasa qui fait suite à l'accident cérébral. L'aphasie qui frappe son personnage et l'impossibilité dans laquelle elle se trouve de communiquer avec ceux qui l'entourent renforcent l'impression de coupure face au monde extérieur. Lors de cette claustration, le physique, le physiologique, l'organique prennent une place centrale. La focalisation se fait sur le corps réduit à ses fonctions vitales, et, même après la phase de réveil, à ses sens, à travers des perceptions basiques telles que le chaud et le froid. "Je sens, donc je suis », tel est le champ de conscience mis en scène.

Pour qualifier le corps « devenu pour le sujet une instance externe/interne, un Moi/nonMoi inquiétant voire répulsif et haï ${ }^{11}$, Isabelle Lasvergnas utilise la métaphore du " corps exorbité ». Corps " exorbité » en effet, parce que tronqué, devenu étranger à luimême : comment reconnaître comme sien ce visage, paralysé, auquel l'on ne parvient plus à insuffler les expressions que l'on désire, ces cheveux rasés d'un côté, dont les repousses sont désormais grises, ce bras qui ne se soulève plus, ces jambes inertes? Corps devenu incontrôlable, dont les incontinences sont relatées de manière extrêmement crue, tandis que la bave qui s'écoule d'une bouche que l'on ne parvient pas à fermer est un leitmotiv présent jusque dans les dernières pages du roman.

$K$. Schmidt dépeint de manière frappante comment son alter ego se retrouve au stade du nouveau-né qui doit ré-apprendre les gestes les plus élémentaires : aller aux toilettes, faire sa toilette, prendre son petit déjeuner, se déplacer à l'aide d'un fauteuil roulant, puis d'un déambulateur, parler, classer, compter, abstraire. Son impuissance est rendue par la multiplication des formes passives: Helene est alimentée, lavée, posée, poussée - par d'autres. L'état végétatif dans lequel elle se trouve d'abord et sa faiblesse physique ensuite semblent par ailleurs la vouer à n'être considérée que comme un objet : on parle d'elle comme si elle n'était pas présente et on la manipule sans prendre garde à sa résistance, ce qui provoque une indicible détresse. Faute de mots pour l'exprimer, elle se saisit du monde avec son corps. Surinvesti émotionnellement, ce dernier parle son propre langage : 
L'enfant se tort, se débat et donne des coups autour de lui. Son père sourit et le tient fermement. Elle n'arrive pas à exprimer ce qu'elle pense. C'est terrible, et c'est elle qui se tort. Qui donne des coups autour d'elle. (80)

Peu à peu les sensations brutes font place aux ressentis, c'est le dégel des affects et la palette des sentiments s'élargit : après l'angoisse, la dépression, la rage, l'humiliation, la frustration et la résignation réapparaissent la plénitude, l'optimisme et même l'euphorie en contrepoint, sans jamais cependant qu'il y ait psychologisation ou que les causes et les conséquences soient véritablement sondées. Cette absence d'intellectualisation est plausible : elle reflète la perte cognitive subie, qui veut que l'on ressente, par exemple, la peur, qui participe de l'instinct de survie, mais pas la tristesse, qui demande une capacité d'abstraction plus importante. Une autre explication à la distance que la patiente semble entretenir face à son propre corps souffrant est la part de déni probablement nécessaire afin d'amortir le choc émotionnel lié à son nouvel état. De même que la douleur physique est masquée par la morphine, l'ignorance protège au moment où il est encore trop tôt pour accepter l'inconcevable. Tard dans le roman, Helene préfère encore ne pas identifier trop précisément le mal qui sommeille en elle : «Elle ne sait pas vraiment $C E$ que c'est, mais décide de s'en accommoder. » (91)

16 K. Schmidt entre dans l'intime de la maladie en pratiquant l'économie des mots et la sobriété des sentiments. La narration est sur le fil, elle évite le pathos et la sensiblerie. Évoluant avec dignité et pudeur, maniant parfois l'humour, elle dit les choses sans jamais se donner en spectacle, ni verser dans la victimisation ou le voyeurisme. Le texte livré est néanmoins douloureux :

Le langage est un animal endormi. Elle doit débusquer chaque mot et le prononcer doucement avant de le faire à haute voix. Mais à présent, tout s'est évanoui, c'est la confusion dans sa tête, même l'animal endormi a disparu [...]. (78)

17 La difficulté à suivre une conversation rapide ou à saisir des énoncés complexes, à supporter qu'il y ait beaucoup de monde dans une pièce, la fréquence de ses oublis, et surtout les mots qui la désertent ou ne franchissent plus ses lèvres à la moindre émotion sont la manifestation des capacités sensiblement réduites de l'esprit subtil que fut un jour Helene. De ce point de vue Du stirbst nicht est à la fois le livre de la renaissance et du deuil: celui de la femme qu'elle était, pour elle-même et les autres, et dont il faut accepter de se séparer.

18 À bien des égards, l'identité de genre d'Helene a été arasée par l'expérience de l'accident. Cette dernière la réduit en effet durant un temps à un "étant-corps-souffrant $»^{12}$, neutre et sans sexe, dont la toilette intime est indifféremment effectuée par des soignants homme ou femme. Elle réapparaît toutefois dès lors que la mémoire se remet en marche. La façon dont Helene recompose mentalement sa famille est particulièrement révélatrice. À un moment où elle semble incapable d'éprouver de la tristesse pour elle-même, le souvenir du suicide de Mischa, fils aîné de son mari Matthes, réveille ses affects et provoque ses pleurs. Par le biais de la mémoire affective liés aux êtres chers, le corps anesthésié, vécu comme un étranger va petit à petit à petit se reconnecter à son monde interne. Helene renoue alors avec ses identités sexuelles et sociales de femme et de mère et trouve à nouveau un ancrage dans l'espace et le temps - dimensions qu'il va toutefois aussi lui falloir reconquérir. 


\section{Amnésie : failles, effritements et traumas collectifs} RDA vient s'ajouter à celle de l'époque contemporaine avec pour charnière l'année-clé 1989. L'histoire individuelle vient ainsi s'imbriquer dans la grande Histoire, la dimension personnelle rejoint l'échelle collective, conférant à $\mathrm{Du}$ stirbst nicht une fonction d'anamnèse qui va au-delà du seul cas de la patiente Helene Wesendahl. Les vignettes biographiques, très souvent consacrées au couple et à la famille, esquissent la trajectoire paradigmatique d'une Allemande de l'Est appartenant à la génération des « Nés dedans » ( Hineingeborene), celle d'après 1945 qui n'a connu que le socialisme réellement existant de RDA. Au gré des souvenirs qui émergent se dessine ainsi en creux la silhouette d'une société socialiste dont les mythes fondateurs sont confrontés au souvenir de la vie au quotidien. Or cet exercice de mémoire tend, de fait, à révéler les failles du système.

Helene rencontre Matthes alors qu'ils ont une vingtaine d'année. Il est déjà marié et père de deux enfants. Elle-même élève seule les deux garçons qu'elle a eus hors mariage, tout en poursuivant son activité professionnelle. De manière emblématique, la recomposition de la famille à la façon d'un puzzle dont les pièces s'imbriquent peu à peu les unes dans les autres correspond au type de foyer qu'Helene et les siens forment, à savoir une famille «recomposée » au sens sociologique du terme. Cette situation correspond à la réalité sociale de la RDA. Elle est rendue possible par l'indépendance financière des femmes, massivement intégrées au monde du travail dès l'après-guerre : parce qu'elles ne sont pas menacées dans leur emploi, et donc fragilisées économiquement, en raison de leur maternité, les femmes peuvent avoir des enfants sans être mariées et divorcer même quand elles sont mères..$^{15}$ Or ce modèle est impensable à l'Ouest, à la même époque et plus tard également: en 1976, pour pouvoir travailler à plein-temps, les mères de famille doivent encore présenter l'autorisation écrite de leur mari, et ce dernier demeure légalement le « chef de famille » jusqu’à nos jours. Par ailleurs, la planification familiale,

Transtext(e)s Transcultures 跨文本跨文化, 11 | 2016 
grâce à l'accès à la pilule et à l'avortement à partir de 1972, assure la bonne santé démographique de la RDA. ${ }^{16}$ Sans doute cela explique-t-il que la pression sur la cellule familiale traditionnelle, et son maintien coûte que coûte, soit moins forte qu'ailleurs, si bien qu'on assiste à de réelles évolutions dans les schémas familiaux. Ces manifestations de l'émancipation des femmes n'empêchent toutefois pas qu'elles soient thématisées dans toute leur complexité et avec leurs paradoxes par K. Schmidt : Matthes doit se battre pour continuer à voir ses deux enfants après son divorce et Helene se résout à réclamer une pension alimentaire au père de ses enfants car ses revenus ne suffisent pas à subvenir aux besoins des siens.

De même, le progressisme juridique n'est pas synonyme d'acceptation sociale. Le régime use de mille détours pour marquer sa désapprobation à l'endroit de l'« adultère » Matthes et de la "traînée » Helene, mère de deux "bâtards », considérés comme des marginaux : on impose à Matthes un emploi sous-qualifié et on leur octroie un appartement exigu, situé à des kilomètres de leur lieu de travail. Devant l'impossibilité d'obtenir un logement suffisamment spacieux (ces derniers sont attribués par l'État), le couple rend publique sa volonté de quitter la RDA (ce qui est impossible en théorie). Ce n'est qu'à ce moment qu'on lui accorde un logement décent - mais à condition qu'il se marie.

On ne peut par ailleurs s'empêcher de voir dans les nombreuses anecdotes évoquant les dysfonctionnements du système de santé est-allemand, pourtant très progressiste et ambitieux à l'origine, une métonymie de l'effritement de l'état socialiste. Ces flash-back ne thématisent du reste pas uniquement le naufrage économique du pays, qui ne parvient pas à venir à bout de la pénurie structurelle qui l'affecte, mais également une forme de désagrégation morale liée au contrôle permanent exercé par le régime sur ses citoyens. La peur des représailles altère durablement les rapports humains et plonge certains habitants dans le désespoir. A contrario, d'autres parviennent à se soustraire à la pression du collectif en mettant en place des stratégies d'accommodement, tandis que les " marges », qui se sont formées aux abords de la société, développent différentes formes de courage civil. Mais quelle que soit l'attitude adoptée, le point commun de nombre de membres de cette génération est la désillusion à l'égard du socialisme réellement existant de RDA, désillusion qui ne laisse aucun doute sur la fragilité d'un système dont les fondements sont sapés. C'est, par exemple, ce qu'Helene retient d'un film, tourné à la fin de la décennie 1980, qui met en scène la jeunesse est-allemande :

Les filles étaient devenues mères très jeunes, les garçons pères. Certains n'avaient pas échappé à la criminalité. Le film n'en était pas pour autant déprimant, mais il rompait définitivement avec les illusions d'antan. Après l'avoir vu, c'en était fini. Et elle avait trouvé cela agréable. (66)

Les passages consacrés à la RDA sont davantage de l'ordre de l'évocation pointilliste que du récit explicatif. Au lecteur non initié, leur contenu pourra même sembler opaque, preuve que K. Schmidt n'entend pas faire preuve de didactisme. Si le roman pointe les maux de la république socialiste, il échappe à tout manichéisme en revenant aussi sur les conséquences biographiques de la césure des années 1990 pour seize millions d'Allemands de l'Est. Une promenade qu'Helene entreprend dans son fauteuil roulant à travers un complexe hospitalier, qu'elle a bien connu du temps de la RDA pour y avoir travaillé, la met face aux cliniques ultramodernes et privées qui se dressent à présent sur le site. La coexistence des anciens et des nouveaux bâtiments évoque en un raccourci saisissant le résultat du passage de l'ancien au nouveau système à présent nommé «Allemagne » et 
face auquel elle affiche une distance ironique : «Là où Est et Ouest s'amalgament pour former une mixture ouestest à partir de l'Est véritable et de l'Ouest réel. » (99)

La chute du mur de Berlin, vécue par cette génération devant son poste de télévision, est présentée en dehors de toute euphorie et de tout sentiment de victoire. Pour ceux qui doivent « reprendre les affaires » (246), c'est la fin de la légèreté. En effet, si la « mixture ouestest " évoquée plus haut a les dehors de l'équilibre, les considérations d'Helene ne laissent pas de doutes sur le rapport de domination qui s'instaure au profit de l'Ouest. À l'Est surviennent bientôt les fermetures de commerces et d'usine, le chômage, la désertification de certaines zones rurales et le déclassement de ses populations, avec pour conséquence de plonger les habitants dans l'alcoolisme et la dépression:

\begin{abstract}
Ils se tenaient avec effroi devant les nombreuses tombes des gens de leur âge (ils en avaient compté dix !), des personnes nées dans les années 1950, plus d'hommes que de femmes, comme si toute une génération avait voulu prendre congé. La boisson avait cogné fort, le bistrot du village se portait à merveille, comme avant [...]. Les hommes qui n'étaient pas en train de boire une bière échangeaient quelques mots avec leur voisin au sujet de la clôture du jardin, on avait l'impression qu'ils s'étaient mis d'accord pour bouger leur lèvres aussi lentement que leurs corps: partout on glandouillait et on traînait les pieds, comme si tout mouvement superflu vous mettait inutilement à contribution. Ils ne se lançaient plus dans de longues discussions, comme si tous avaient à l'esprit une sorte de fin et étaient tétanisés, de peur que cette pensée ne leur échappe. (247)
\end{abstract}

C'est ainsi toute une génération qui tire sa révérence, tandis que se fait jour chez ceux qui restent l'«ostalgie ", ce sentiment mélancolique positif éprouvé rétrospectivement à l'égard de la RDA. Helene le met davantage sur le compte d'un attachement à l'enfance et à la jeunesse qu'à une réelle idéalisation de l'État est-allemand ou à un repli identitaire. Elle y reste pour sa part hermétique. L'épiphanie espérée après l'achat d'une marque de chocolat est-allemand à nouveau disponible dans le commerce ne se produit pas chez elle.

La chronique de la maladie d'Helene Wesendahl par K. Schmidt possède bien le « double mouvement d'hyper présence et de distanciation» caractéristique de l'«art dépositionnel $\aleph^{17}$. L'on connaît par ailleurs les effets cathartiques et curateurs du storytelling pour les traumatisés de l'histoire, ainsi que la fonction de contrepoint de ces récits face aux récits dominants..$^{18}$ L'auteure propose par le biais de son personnage, un bilan contrasté et apaisé de l'avant et de l'après 1989, sans langue de bois, mais sans non plus adopter le ton du règlement de compte :

Tout est relatif, se dit-elle à présent. À chaque chose est attribué un petit bout de jardin historique sur lequel il lui faut tenir, et lorsqu'on n'est plus en mesure d'exploiter cette parcelle, il faut céder la place. (108)

La narration de K. Schmidt doit être interprétée comme un complément, un pendant subjectif et pertinent sur le plan psychologique, une histoire d'en bas face à l'histoire officielle, plus que comme une contestation. Ceci est d'autant plus vrai que l'exercice mémoriel contribue aussi à faire émerger des réflexions inédites chez Helene. Vaincre l'aphasie et l'amnésie, c'est parvenir à utiliser le mot juste afin de nommer les choses, reconstituer les événements au plus près du réel - or ce travail possède également une dimension productive : il donne lieu à de nouvelles visions et à des réinterprétations de ce qui a été : «La façon dont ses opinions, qu'elle croyait fermement établies, se croisent et se reconfigurent d'une façon totalement différente de celle du passé est surprenante » 
(101). C'est à ce réagencement des constellations et des événements, qui à la fois oblige et offre la possibilité de resignifier les choses, que nous allons à présent nous intéresser en nous penchant sur le thème des amours et de l'écriture sous l'angle du trouble et de la métamorphose.

\section{Cure : narration queer et écriture hybride}

Du stirbst nicht accorde une grande importance aux amours de son personnage principal, le sentiment amoureux étant lui aussi rapporté à une sensation physique : «Où en étaitelle restée ? À l'amour. Un sentiment. Qui fait s'accélérer le sang. Quand elle pense à l'amour, elle pense à chaud et rouge » (49). Si la relation avec Matthes est d'un côté placée sous le signe du soutien, de la prévenance et du dévouement, de l'autre, l'impression que quelque chose détone affleure dès le réveil : «Pas de tiraillement. C'était pourtant le cas avant. Elle le sait bien» (39), tandis que des questions sèment le doute: "Pourquoi devrait-elle l'aimer? Elle l'a tout simplement oublié» (137). Ce que sa mémoire altérée refuse de lui révéler mais que son corps lui souffle avec insistance lui est confirmé par la littérature: la lecture du texte schwerwasser.doc, l'un des derniers qu'elle ait écrits, retrouvé dans son ordinateur portable, lui apprend que son malaise est justifié. Lorsqu'enfin, à la faveur d'une réminiscence, elle visualise une liste d'affaires à emporter épinglée sur le frigo, le stress et la douleur éprouvés sont un écho à ceux ressentis lors d'une infidélité de Matthes 16 ans plus tôt. Par cet effet miroir, elle comprend qu'elle s'apprêtait à se séparer de son mari.

Le trouble d'Helene est dû aux sentiments qu'elle éprouve envers Viola. Dans ce cas encore, c'est grâce à la mémoire du corps qu'ont lieu les retrouvailles :

Tandis qu'elle réfléchit, elle a la sensation que quelqu'un vient s'appuyer contre son dos. Un quelqu'un dont les os sont pointus. Elle sent même sa tête et ses cheveux longs qui à présent tombent sur ses épaules, ils sont grisonnants, ce quelqu'un n'est donc plus tout jeune. [...] Il passe à présent sa main par-dessus sa tête et la sienne et la pose sur son front. Cette caresse la fait trembler, elle sent parfaitement sa douce pression, jusque sur les os rendus insensibles par l'opération. (125)

Helene se love dès lors dans le souvenir de l'apparition changeante, tantôt douce, tantôt fougueuse, de Viola - alias Victor Malysch. Cette ombre surgie du passé, synonyme de mystère et de facétie, vient placer les amours hétérosexuelles et somme toute fort conventionnelles d'Helene sous le signe de la surprise et de l'étrange, y compris pour ellemême : « Avant-hier encore, elle aurait sans doute été politiquement incorrecte en riant à la pensée d'avoir aimé une femme. Comme tout est instable à présent, encore et encore " (138). En mettant en scène le personnage transsexuel de Viola (sans jamais le qualifier ainsi), le roman creuse la veine thématique de la mue et de la métamorphose tout en la radicalisant. Parce qu'il tend à interroger et à problématiser la bicatégorisation traditionnelle entre hommes et femmes des sexes et des genres, ce récit parallèle à l'histoire d'amour conjugal peut être qualifié de queer. Il thématise, en les variant, l'incertitude et la déstabilisation fondamentales dans lesquelles Helene vit depuis son accident.

Vient alors s'enchâsser dans le processus de remémoration de son propre passé la biographie de Victor/Viola, depuis son mariage avec une femme convaincue d'épouser un 
homme - ce qui correspond à son sexe biologique, mais non à son identité psychologique jusqu'à sa mort en juin de la même année. Celle-ci se fait à nouveau par l'intermédiaire d'une série de réminiscences et d'informations partielles, échappant à la chronologie : le souvenir d'un baiser, une pince à cheveux retrouvée, les questions de son amie Carla ou encore les mails stockés dans son ordinateur. Outre les souvenirs, aléatoires et hétérogènes, le caractère changeant de Viola se traduit dans les portraits que nous en livre Helene qui, à chaque rencontre, fait face à une nouvelle personne : être vêtu de manière neutre lors du premier rendez-vous au château Sans-Souci de Potsdam, vamp ultra maquillée lors d'un week-end à Karlshorst, femme aux cheveux courts, androgyne et plus attirante que jamais dans un bar de Berlin. Or c'est précisément cette ambivalence qui séduit Helene. Ainsi utilise-t-elle presque systématiquement le surnom «Maljutka Malysch » (148) dans ses évocations, la " petite bonhomme » en russe, terme qui reprend et célèbre cette ambiguïté.

La remémoration d'Helene se veut aussi commémoration. Comme une miniature, la clinique de la transformation de Victor en Viola s'imbrique dans celle de sa propre convalescence, en peignant un autre type de parcours de souffrance. Cette dernière est d'abord d'ordre physique. Le roman aborde très concrètement les effets secondaires liés à la prise d'hormones: le poids, la couperose qu'il faut soigner à l'aide de crèmes et qui réapparaît à chaque rasage. Elle évoque les contraintes que Viola s'impose afin de ressembler à une femme et enfin la mutilation de son sexe suite à la chirurgie. Les embûches d'ordre économique et social ne sont pas moindres. Viola perd tour à tour sa femme, ses amis, ses différents gagne-pain, ses loisirs et voit inexorablement son univers se rétrécir.

Le changement de sexe vu à travers les yeux d'Helene est incontestablement une violence que Viola s'inflige à première vue volontairement, mais que la société rend obligatoire dans la mesure où elle est incapable de penser l'identité en dehors des catégories binaires d'homme et de femme. Pour être considérée comme une femme, il faut se donner à voir et à entendre comme telle. Ceci explique que la difficulté à être soi demeure après l'intervention: «Ce n'est que lorsqu'elles se mirent à parler du passé, de ses études, de son service militaire et, en effet, de son mariage, que Viola se mit à dire haut et fort je, comme si elle n'était pas du tout certaine de son moi présent». Isolée, précarisée, soumise à une énorme pression psychique, elle sombre dans la boisson jusqu'à son décès solitaire un jour de juin. Auparavant elle vit néanmoins le bonheur d'avoir renoué avec ses deux fils.

36 «À l'opposé de l'amour qui change le corps en chair, la mort est ce qui change la chair en corps $»^{19}$, nous dit Camille Laurens. Ainsi les sentiments d'Helene permettent-ils au corps violenté et mutilé de Viola de renaître à l'amour, tandis qu'après sa disparition, la narration de leur relation l'arrache au néant. En disant le corps manquant, le corps qui se tait désormais, elle permet l'identification à ce corps, elle en inscrit la trace et lui offre un lieu d'inhumation dans la mémoire collective..$^{20}$ Or ce processus possède une dimension réparatrice qui va au-delà de la simple possibilité de faire son deuil.

En mettant en pensées et en mots la matrice informe que sont devenus pour elle les souvenirs et le langage, Helene vainc petit à petit l'aphasie et la perte cognitive qui l'accablent. Le recouvrement de ses facultés intellectuelles, la capacité retrouvée à jongler et à inventer se traduisent dans des passages qui réunissent fond et forme sous le signe de la transformation et de l'hybride, comme lorsqu'Helene et Viola s'opposent dans un pingpong de mots « bifrons » (Janusworte), à la fois homonymes et antonymes, ou qu'Helene, 
écartelée entre Matthes et Viola, assemble, à l'instar de certains jeux d'enfants, les différentes parties de leur corps pour en créer un autre: «Durant un temps, elle est tellement déconcertée qu'elle voit Matthes avec le visage de Maljutka, Maljutka avec celui de Matthes, leurs silhouettes se confondent, n'en font plus qu'une pour enfin se séparer. Heureusement chacune avec sa propre tête. » (202)

Par une sorte d'effet de contamination, les remaniements en cours dans la vision qu'Helene a des êtres, mais aussi dans sa conception des choses, semblent imprégner le matériau langagier au moment où ce dernier se redonne à elle :

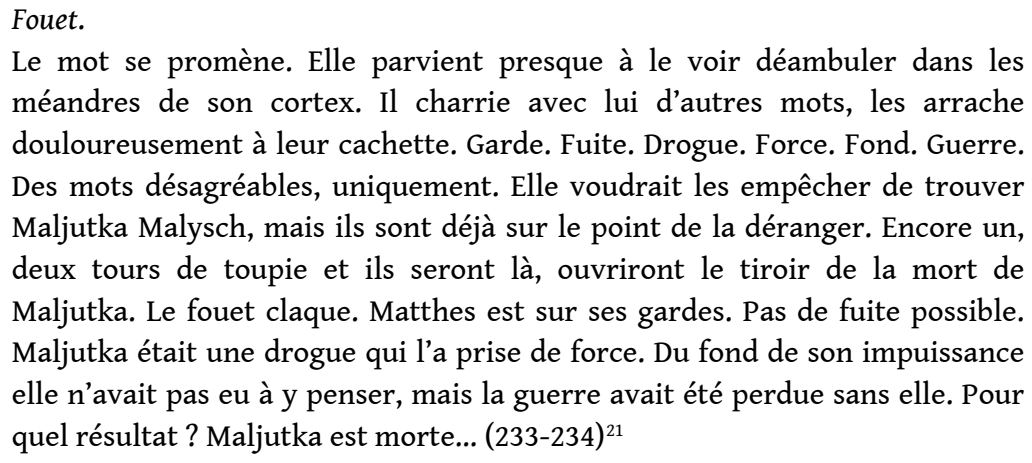

Alors que quelques pages plus tôt, Helene faisait ses adieux à la poésie sonore de Friederike Mayröcker et d'Ernst Jandl, pensant qu'elle ne pourrait plus jamais la lire à haute voix, et doutait fortement de sa propre capacité à réécrire un jour des poèmes, les associations, les jeux de mots et de sonorités, les rapprochements ludiques, même quand ils sont douloureux, réapparaissent. Ils confèrent au texte en prose la fluidité et la plasticité de la poésie. Et en font rebattre le pouls comme par le passé :

Elle se souvient avoir éprouvé autrefois des sensations physiques en lisant ou en entendant de bons poèmes. Ils étaient généralement accompagnés de sensations intellectuelles indescriptibles. Elle était totalement excitée, dans un état second, les mots déclenchaient à eux-seuls une avalanche dans sa tête, si bien qu'elle n'avait plus qu'à écrire ce qui lui venait à l'esprit. (250)

Plus que jamais, l'écriture est présentée comme une opération de chair, traversée, incarnée par le souffle et le flux de la langue. C'est ici que la fécondation de l'écriture, son hybridation fructueuse entre prose et poésie par le truchement du corps sont les plus frappantes. Juste derrière Helene se tient naturellement Kathrin Schmidt qui, avec $D u$ stirbst nicht, surmonte le trauma physique massif qu'elle a subi par l'acte créatif. La parution du recueil de poésie Blinde Bienen en 2010 est une autre magnifique preuve que les histoires ne finissent pas toujours mal.

41 Si Du stirbst nicht est un ouvrage qui met en scène différentes transitions dans la vie de son personnage principal après 1989 , le traitement esthétique de l'accident cérébral proposé par son auteure marque quant à lui une évolution pour la littérature d'Allemagne de l'Est dans son ensemble. La subjectivité, honnie par le réalisme socialiste pour qui seuls le "typique » et l'« exemplaire » avaient droit de cité, avait certes déjà été remise à l'honneur par des auteures de la génération dite «intermédiaire », telles que Brigitte Reimann (1933-1973), Maxie Wander (1933-1977) et naturellement Christa Wolf (1929-2011) - mais sans jamais que leur réception de la modernité ne s'aventure ouvertement sur les terres de l'autofiction. 
42 À l'occasion de circonstances biographiques dramatiques, Schmidt surmonte ses réticences envers ce genre et dépasse la détresse consistant « à être privée de sa vie, tout en devant rester en vie » (289) en recourant à l'écriture de soi. ${ }^{22}$ Laissant le pathogène faire irruption dans son œuvre et en influencer la dynamique interne, elle fait la preuve que non seulement le corps, mais de plus le corps souffrant, montré dans ses fonctions les plus triviales, peut être raconté avec pudeur.

$43 \mathrm{~K}$. Schmidt ne renonce pas ce faisant à la dimension sociale de l'écriture. Interpréter le trauma causé par l'accident cérébral à son personnage comme une métaphore du trauma collectif lié à la chute du Mur serait sans doute téméraire, de même que son mauvais état de santé ne reflète pas stricto sensu le mauvais fonctionnement des systèmes ou les difficultés de la population. On peut néanmoins voir dans son travail d'anamnèse, y compris au sujet des maux collectifs de la RDA et de l'Allemagne réunifiée, l'intention testimoniale qui est le propre des individus ayant subi un choc émotionnel profond.

Qu'en est-il des questions relevant de la problématique du genre posées en introduction? Le passage en revue des années précédant 1989 prouve que la RDA demeure un pays où l'on se trompe, où l'on est jaloux, où les chefs séduisent leurs subordonnées, où l'on n'a pas exactement la même relation à ses fils et ses filles selon que l'on soit mère ou père, où, en résumé, ni les rapports hommes-femmes, ni les modèles traditionnels de ce que doit être un couple n'ont totalement changé. Toutefois, si le mythe de l'égalité homme femme véhiculé par le discours officiel est quelque peu écorné, force est de constater que les principaux protagonistes féminin et masculin échappent largement aux stéréotypes de genre, de même que les conflits au sein du couple n'ont pas leur source dans une infériorisation de la femme ou dans un mauvais équilibre entre partenaires, mais dans la contradiction des sentiments et les errements du cœur. Et si une forme de domination est dénoncée, c'est bien plus celle qui oppose le pouvoir aux citoyens. Il est difficile de savoir si cette absence de thématisation est le reflet d'une occultation de la problématique ou, au contraire, la preuve d'un degré d'émancipation (plus) important chez les Allemand(e)s de l'Est. Là où en revanche, le roman sort des sentiers battus, c'est lorsqu'il choisit un personnage transsexuel pour incarner le nouvel amour d'Helene. La présentation de sa trajectoire contribue à questionner les identités bicatégorielles et hétéronormées majoritaires - sans pour autant invalider ou déconstruire ces modèles. Que cette partition soit jouée par une mère de famille à la vie relativement conventionnelle n'en est que plus fort.

Sans doute peut-on parler de point de vue féminin si l'on tient compte de l'importance de la cellule familiale, de la place accordée aux grossesses, de la culpabilité de mère qui tarde à se souvenir de sa petite fille à son réveil, du rôle central que joue l'amour dans la narration. Faut-il pour autant voir dans $\mathrm{Du}$ stirbst nicht une expression de l'« écriture féminine »? Ce serait sans doute une vision trop réductrice. On en est encore moins convaincu lorsqu'on considère que les thèmes de l'amour, de la parentalité, du deuil d'un enfant, de la maladie et de la mort sont traités depuis fort longtemps sur le même mode intimiste par les maîtres de l'autofiction que sont en France, par exemple, Philippe Forest dans l'Enfant éternel (1997) ou Emmanuel Carrère dans D'autres vie que la mienne (2009). Sans doute faudrait-il, pour aller plus loin, affiner cette perception en procédant à une analyse comparative qui s'attacherait aux caractéristiques formelles et stylistiques mises en œuvre par les auteurs et les auteures. 


\section{NOTES}

1. Pour un rappel complet des développements et évolutions de la question de l'«écriture féminine ", depuis les perceptions les moins élaborées (plus de psychologie, motifs spécifiques, moins de cruauté et de brutalité, style plus lisse et simple), jusqu'aux travaux de Sigrid Weigel ( Die Stimme der Medusa, 1987) qui postulent le regard «dédoublé » à la fois sur soi et le monde, le jeu et la déconstruction des modèles de genre, la disparition de la frontière entre monde intérieur et extérieur ainsi que la subversion de l'ordre logo- et phallocentrique, voir l'ouvrage de Nicole Masanek, Männliches und weibliches Schreiben? Zur Konstruktion und Subversion in der Literatur, Würzburg, Könighausen \& Neumann, 2005, p. 7-53.

2. Pour une présentation de la condition de la femme chez les penseurs socialistes, voir, par exemple, Herta Kuhrig, Wulfram Speigner (dir.), Wie emanzipert sind die Frauen in der DDR?, Cologne, Pahl Rugenstein Verlag, 1979, p.10-13 et plus récemment Grit Bühler, Mythos Gleichberechtigung in der DDR. Politische Partizipation von Frauen am Beispiel des Demokratischen Frauenbunds Deutschlands, Francfort/Main, Campus Verlag, 1997, p. 16.

3. Anna Kaminsky, Frauen in der DDR, Berlin, Christoph Links, 2016, p. 16-18.

4. Sandrine Kott, Françoise Thébaud (dir.), «Éditorial », Clio, 1/2015 ( ${ }^{\circ} 41$ ), p. 7.URL : http://www.cairn.info/revue-clio-femmes-genre-histoire-2015-1-page-7.htm. Dernière consultation : 4.5.2017.

5. Cela étant, l'anévrisme intracérébral touche en France une fois et demie plus de femmes âgées de 35 à 60 ans que d'hommes en moyenne, tandis que l'anévrisme de l'aorte abdominale concerne majoritairement les hommes âgés de plus de 50 ans. Source : http:// www.santemagazine.fr/prevenir-la-rupture-d-anevrisme-29577.html. Dernière consultation: 7 avril 2017. La tendance est la même en Allemagne. Source: http:// www.spiegel.de/gesundheit/diagnose/erweiterte-blutgefaesse-aneurysma-in-bauchoder-kopf-a-874141.html. Dernière consultation : 4.5.2017.

6. De récents travaux étudient la construction de la différence entre les genres dans la littérature d'Europe de l'Ouest du XVIIIe au XXe siècle, en particulier en Allemagne. Les contributions montrent, par exemple, comment les romans de formation écrits par des femmes au XIXe siècle thématisent l'absence d'émancipation de leurs héroïnes et leur non-reconnaissance par la société par le biais de la maladie. C'est la même perspective qui se retrouve au XXe siècle dans des narrations de Gabriele Reuter et Elfriede Jelinek, entre autres. Celles-ci dénoncent un monde et un système de valeurs qui pathologisent les femmes afin de les priver de leur liberté. Rudolf Kaiser, Beate Schappach (dir.), Krank geschrieben. Gesundheit und Krankheit im Diskursfeld von Literatur, Geschlecht und Medizin, Bielefeld, transcript, 2014.

7. La définition de l'autofiction reprise ici est la suivante: «[...] j'ai tenté de définir ce genre par sa stratégie pragmatique de double affichage d'indices contradictoires. Et j'ai suggéré que cette ambivalence traduit une incertitude fondamentale quant à l'identité du personnage, donc, en dernière analyse de l'auteur. " Philippe Gasparini, Autofiction. Une aventure du langage, Seuil, Paris, 2008, p. 46. En 1977, Serge Doubrovsky avait forgé le terme en le définissant ainsi : "Autobiographie? Non. C'est un privilège réservé aux importants de ce monde au soir de leur vie et dans un beau style. Fiction d'événements et de faits strictement réels ; si l'on veut autofiction, d'avoir confié le langage d'une aventure 
à l'aventure du langage, hors sagesse et hors syntaxe du roman, traditionnel ou nouveau. », Ibid., p. 15.

8. Sibylle Goepper, « Du sujet lyrique au sujet autofictionnel. La scène littéraire berlinoise à l'épreuve du (tournant > ", in Clara Royer, Petra James (dir.), Sans faucille ni marteau. Ruptures et retours dans les littératures européennes post-communistes, Bruxelles, Peter Lang, 2013, p. 67-81.

9. Thierry Groensteen est cité dans Lilian Cheilan, «Ce que dit le corps dans la bande dessinée autobiographique. Quelques exemples, in Jean-François Chiantaretto, Catherine Matha (dir.), Écritures de soi, Écritures du corps, Paris, éditions Hermann, 2016, p. 85.

10. Toutes les citations sont traduites par nos soins.

11. Isabelle Lasvergnas, «Le corps exorbité », in J.-F. Chiantaretto, C. Matha, Écritures de soi, op. cit., p. 117.

12. Ibid., p. 118.

13. Camille Laurens, «Le pouls de la langue », in J.-F. Chiantaretto, C. Matha, Écritures de soi, op. cit., p. 31-32.

14. Dori Laub, «Un événement sans témoin: vérité, témoignage et survie », in «Dori Laub. Une clinique de l'extrême", Le Coq-héron, 1/2015 (n²20), p. 83-94. URL: http:// www.cairn.info/revue-le-coq-heron-2015-1-page-83.htm. Dernière consultation : 4.5.2017

15. Voir les développements sur le divorce en RDA in A. Kaminsky, Frauen in der DDR, op. cit., p. 160-166.

16. Wolfgang Weiss, « Aspects démographiques régionaux de la fusion allemande. Un bilan vingt ans plus tard », in Anne-Marie Pailhès, Jacques Poumet (dir.), « Vivre en Allemagne de l'Est, vingt ans après la chute du mur » (dossier), Allemagne d'aujourd'hui, $\mathrm{n}^{\circ}$ 190, 2009. Merci à Anne-Marie Pailhès pour cette référence.

17. L'expression est empruntée à Dori Laub et Aharon Appelfeld par Françoise Davoine, in «Introduction », « Dori Laub. Une clinique de l'extrême», Le Coq-héron, op. cit., p. 9-12. URL : http://www.cairn.info/revue-le-coq-heron-2015-1-page-9.htm. Dernière consultation : 4.5.2017.

18. Fabrice Mourlon, «La place du corps dans les récits de survivants du conflit nordirlandais ", in J.-F. Chiantaretto, C. Matha, Écritures de soi, op. cit., p. 272. Ce point de vue est toutefois relativisé par certaines victimes des camps. Ainsi Jorge Semprun déclare-t-il avoir renoncé à écrire son expérience de Buchenwald pendant 17 ans pour pouvoir survivre. D'après lui, le retour vers cette mémoire traumatique l'aurait conduit au suicide d'où la mise en pratique d'« une sorte de thérapie systématique, parfois brutale, de l'oubli ». In : http://www.gallimard.fr/catalog/entretiens/01029405.htm. Merci à Marie Rosier pour cette référence.

19. C. Laurens, «Le pouls de la langue », op. cit., p. 28.

20. Janine Altounian, «Lors d'un héritage traumatique, l'écriture de soi inscrit implicitement des corps absents, violentés ou en détresse ", in J.-F. Chiantaretto, C. Matha, Écritures de soi, op. cit., p. 283.

21. Les mots employés par Helene en allemand sont tous monosyllabiques et composés sur la même allitération cht: Wucht, Wacht, Flucht, Sucht, Kraft, Schlucht, Schlacht.

22. Kathrin Schmidt, «Ich fand ganz viele Texte, die zwar nicht die Wende als politischen Akt zum Anlaß nahmen, aber dennoch ein Reflex darauf waren ", in Sibylle Goepper, 
Cécile Millot (dir.), Lyrik nach 1989. Gewendete Lyrik?, Halle, Mitteldeutscher Verlag, 2016, p. 441.

\section{ABSTRACTS}

Le roman Du stirbst nicht de Kathrin Schmidt met en scène le retour à la vie d'Helene Wesendahl, victime d'un accident cérébral à l'âge de 44 ans, dont on comprend aisément qu'elle est l'alter ego de l'auteure. Outre la chronique de son réveil et de sa convalescence physique, le combat contre les séquelles de la pathologie (aphasie, amnésie), il décrit le processus de reconstruction mémoriel de son passé, immédiat et plus lointain. Le genre permet d'apporter une clé de lecture supplémentaire à ce travail de remémoration qui revient sur la trajectoire de citoyenne d'Helene en République démocratique allemande (RDA) et sur ses amours troublées entre son mari Matthes et son amante Viola. Le récit individuel se double d'une dimension collective portant sur les maux de la république révolue et de l'Allemagne unifiée actuelle. Au croisement de ces différentes strates surgit pour finir le potentiel de résilience de l'expérience narrée qui donne l'occasion à la patiente de reconfigurer de façon inattendue le langage et les significations d'antan. La maladie impacte ainsi tout à la fois le réel, en modifiant sa vision des rapports de couple et des catégories de genre, et l'écriture sur le plan thématique et stylistique.

Kathrin Schmidt's novel called Du stirbst nicht features Helene Wesendahl's return to life; the latter suffered a stroke at the age of 44 and we easily understand that she is the author's alter ego. In addition to the chronicle of her awakening and her physical recovery, the struggle against the pathology's consequences (aphasia, amnesia), it describes the process of memory reconstruction of her immediate or distant past. Gender provides an additional key to understanding the recalling work of Helene which comes back to her path as a citizen in the (GDR) and to her complicated relationship to Matthes, her husband, and Viola, her lover. The individual story is coupled with a collective dimension of the ills of the republic that has gone and with the current reunited Germany. Lastly, at the crossroads of these different strata, the capacity for resilience arises thanks to the recounted experience. It gives the patient the opportunity to reconfigure language and former meanings in an unexpected way. Thus, the disease has at the same time an impact on the reality by altering Helene's vision both of the category of couple and gender and her writing in terms of topic and style.

\section{AUTHOR}

\section{SIBYLLE GOEPPER}

Sibylle Goepper est maîtresse de conférences en études germaniques à l'Université Jean Moulin Lyon 3. Sa recherche porte sur le rapport entre artistes et pouvoir, ainsi que sur les phénomènes de marginalité, de subculture et de dissidence en RDA. Elle s'intéresse également aux évolutions de la littérature dans l'Allemagne réunifiée, en particulier aux écritures autobiographiques et autofictionnelles. Depuis quelques années, elle utilise les outils du genre afin d'appréhender ces différents corpus.

Sibylle Goepper is senior lecturer in German studies at the University of Jean Moulin Lyon 3. Her work is about the relationship between artists and power as well as phenomena of marginality, 
subculture and dissidence within the German Democratic Republic (GDR). She also focuses on evolutions of literature in reunited Germany especially on autobiographical and auto-fictional forms of writing. She has been using gender tools for years as to grasp these different corpuses. 